\title{
07.1
}

\section{Роль кулоновского взаимодействия в дефектной модели барьера Шоттки}

\author{
() С.Ю. Давыдов ${ }^{1}$, О.В. Посредник ${ }^{2}$ \\ ${ }^{1}$ Физико-технический институт им. А.Ф. Иофрфе РАН, \\ Санкт-Петербург, Россия \\ ${ }^{2}$ Санкт-Петербургский государственный электротехнический университет „ЛЭТИ“, \\ Санкт-Петербург, Россия \\ E-mail: Sergei_Davydov@mail.ru
}

Поступило в Редакцию 23 октября 2020 г.

В окончательной редакции 21 ноября 2020 г.

Принято к публикации 22 ноября 2020 г.

\begin{abstract}
Предложена модель барьера Шоттки, в которой на интерфейсе присутствуют димеры, состоящие из полупроводниковых дефектов и ближайших к ним атомов металла. Между электронами дефектов и металлических атомов включено короткодействующее кулоновское отталкивание. Получены аналитические выражения для чисел заполнения атомов и дефектов и высоты барьера Шоттки.
\end{abstract}

Ключевые слова: интерфейсный полупроводниковый дефект, интерфейсный атом металла, кулоновское отталкивание, числа заполнения.

DOI: 10.21883/PJTF.2021.05.50673.18594

Задача о расчете высоты барьера Шоттки (БШ), несмотря на свой ,почтенный возраст“, по-прежнему является актуальной [1-3]. Более того, в послеграфеновый период естественным образом возник интерес к БШ в низкоразмерных структурах (см., например, работы [4-8] и ссылки, приведенные в них). В трехмерном случае существуют различные схемы расчета высоты БШ и различные модели контакта металл-полупроводник, среди которых довольно популярна дефектная модель [1-3]. В работах $[9,10]$ была предложена простая схема расчета высоты БШ на контакте металл-политип $\mathrm{SiC}$, основанная на модификации дефектной модели, предложенной в работах $[11,12]$ и базирующейся на теории адсорбции Андерсона-Ньюнса [13,14]. В основе модели $[11,12]$ лежит допущение, что в задаче о БШ полупроводник можно охарактеризовать локальным дефектным состоянием, лежащим внутри запрещенной зоны. При этом предполагается, что дефекты возникают на начальной стадии формирования интерфейса. В работах $[9,10]$ в качестве дефектов рассматривались вакансии, связанные с присущей политипам $\mathrm{SiC}$ нестехиометричностью $[15,16]$. Эта схема позволила определить характеристики ряда контактов металлов с политипами $\mathrm{SiC}$.

В $[9,10]$ использовался одночастичный подход. В настоящей работе мы рассмотрим кулоновскую связь между металлическими и полупроводниковыми дефектными состояниями на интерфейсе. Насколько известно авторам, в существующих моделях кулоновское взаимодействие электронов металла и полупроводника никогда явно не учитывалось, хотя по аналогии с теорией адсорбции $[13,14]$ логично предположить, что включение такой связи существенно влияет на переход заряда между металлом и полупроводником и, следовательно, на высоту БШ.
Рассмотрим для начала короткодействующее кулоновское отталкивание $G$ электрона дефекта, находящегося в состоянии $|d\rangle$ с энергией $\varepsilon_{d}$, и электрона ближайшего к дефекту атома металла, находящегося в состоянии $|m\rangle$ с энергией $\varepsilon_{m}$. Гамильтониан такой двухуровневой системы

$$
H_{0}=\varepsilon_{m} \hat{n}_{m}+\varepsilon_{d} \hat{n}_{d}+G \hat{n}_{m} \hat{n}_{d}
$$

где $\hat{n}_{m}=c_{m}^{+} c_{m}$ и $\hat{n}_{d}=d^{+} d-$ операторы чисел заполнения атома металла и дефекта, $c_{m}^{+}\left(c_{m}\right)$ и $d^{+}(d)-$ соответствующие операторы рождения (уничтожения). При температуре $T=0$ энергия двухуровневой системы $E_{0}=\varepsilon_{m} n_{m}^{0}+\varepsilon_{d} n_{d}^{0}+G n_{m}^{0} n_{d}^{0}$, где $n_{m}^{0}=\left\langle\hat{n}_{m}\right\rangle, n_{d}^{0}=\left\langle\hat{n}_{d}\right\rangle$; $\langle\ldots\rangle$ означает усреднение по основному состоянию гамильтониана $H_{0}$. Поскольку $n_{m}^{0}+n_{d}^{0}=n^{0}=\mathrm{const}$, ясно, что минимальное значение энергии $E_{0}$ есть $\varepsilon_{m} n^{0}$ при $\varepsilon_{m}<\varepsilon_{d}$ и $\varepsilon_{d} n^{0}$ при $\varepsilon_{m}>\varepsilon_{d}$. Таким образом, учет кулоновского отталкивания ведет к переходу заряда. Отметим, что гамильтониан (1) представляет собой простейший вариант модели Фаликова-Кимболла [17].

Включим теперь взаимодействие $V$ состояний $\varepsilon_{m}$ и $\varepsilon_{d}$ с блоховскими состояниями металла $|k\rangle$. Игнорируя спин, представим соответствующий гамильтониан в виде

$$
H=H_{0}+\sum_{k} \varepsilon_{k} \hat{n}_{k}+V N_{k}^{-1} \sum_{k}\left[c_{k}^{+}\left(c_{m}+d\right)+\left(c_{m}^{+}+d^{+}\right) c_{k}\right] \text {, }
$$

где $\varepsilon_{k}-$ закон дисперсии электронов в металле, $\hat{n}_{k}=c_{k}^{+} c_{k}, c_{k}^{+}\left(c_{k}\right)$ - операторы рождения (уничтожения) электрона металла, $N_{k}-$ число состояний $|k\rangle$ в зоне Бриллюэна металла. В рамках теории Хартри-Фока $[13,14]$ гамильтониан (2) можно преобра- 
зовать к виду

$$
\begin{aligned}
H^{\mathrm{HF}}= & \sum_{k} \varepsilon_{k} \hat{n}_{k}+w_{m} \hat{n}_{m}+w_{d} \hat{n}_{d} \\
& +V N_{k}^{-1} \sum_{k}\left(c_{k}^{+} d+d^{+} c_{k}\right)-G n_{m} n_{d},
\end{aligned}
$$

где $w_{m, d}=\varepsilon_{m, d}+G n_{d, m}, \quad n_{m, d}=\left\langle\hat{n}_{m, d}\right\rangle ;\langle\ldots\rangle$ означает усреднение по основному состоянию гамильтониана (2). Отметим, во-первых, что выделенный атом металла, взаимодействующий с дефектом, рассматривается здесь как адатом. Во-вторых, в (3) мы считаем для простоты, что матричный элемент взаимодействия с металлом $V$ один и тот же для адатома и дефекта, хотя обобщение типа $V_{d} \neq V_{m}$ тривиально.

Легко показать $[18,19]$, что гамильтониану (3) отвечают функции Грина

$$
G_{m, d}(\omega)=\left(\omega-w_{m, d}-\Lambda(\omega)+i \Gamma(\omega)\right)^{-1},
$$

где $\Gamma(\omega)=\pi V^{2} \rho_{m e t}(\omega)$ - полуширина квазиуровней дефекта и адатома металла $\left(\rho_{\text {met }}(\omega)\right.$ - плотность состояний металла), $\Lambda(\omega)$ - гильберт-трансформанта функции $\Gamma(\omega)[13,14]$. Соответствующие функциям Грина (4) плотности состояний имеют вид

$$
\rho_{m, d}(\omega)=\frac{1}{\pi} \frac{\Gamma(\omega)}{\left(\omega-w_{m, d}-\Lambda(\omega)\right)^{2}+\Gamma(\omega)^{2}} .
$$

Для дальнейшего рассмотрения необходимо конкретизировать плотность состояний металла $\rho_{\text {met }}(\omega)$. В простейшем случае (модель Андерсона) можно положить $\rho_{\text {met }}(\omega)=\mathrm{const}$, откуда получаем $\Gamma(\omega)=\Gamma=\mathrm{const}$ и $\Lambda(\omega)=0[13,14]$. Такое приближение приемлемо в том случае, когда перенормированные уровни металлического адатома $w_{m}$ и дефекта $w_{d}$ лежат вдали от дна зоны проводимости металла и $\Gamma \ll W$, где $W-$ ширина зоны проводимости. Подобная ситуация легко реализуется для простых $s p$-металлов (типа $\mathrm{Al}$ ) и переходных металлов с широкой $d$-зоной. Для $\mathrm{Cu}, \mathrm{Ag}$ и $\mathrm{Au}$, имеющих сравнительно узкую $d$-зону [18], больше подходит модель типа „пьедестал“ или модель Фриделя: $\rho_{\text {met }}^{\prime}(\omega)=\rho=\mathrm{const}$ при $|\omega| \leq W / 2$ и $\rho_{\text {met }}(\omega)=0$ при $|\omega|>W / 2$ (за нуль энергии принят центр зоны). Тогда $\Gamma(\omega)=\pi V^{2} \rho=\Gamma=$ const при $|\omega| \leq W / 2, \Gamma(\omega)=0$ при $|\omega|>W / 2$ и $\Lambda(\omega)=(\Gamma / \pi) \ln |(\omega+W / 2) /(\omega-W / 2)|$. Отметим, что в приведенных выкладках предполагается, что „изъятие“ одного поверхностного атома металла не сказывается на плотности состояний $\rho_{\text {met }}(\omega)$.

Оставаясь в рамках простейшей модели Андерсона, при $T=0$ получим систему двух самосогласованных уравнений для чисел заполнения

$$
n_{m, d}=\frac{2}{\pi} \operatorname{arccot} \frac{w_{m, d}-\varepsilon_{\mathrm{F}}}{\Gamma},
$$

где $\varepsilon_{\mathrm{F}}$ - уровень Ферми (множитель 2 появился из-за учета спина). Таким образом, на интерфейсе БШ имеем димер с зарядами $Z_{m, d}=1-n_{m, d}$.
Воспользовавшись результатами [9-12], положение уровня Ферми относительно потолка валентной зоны полупроводника определим из уравнения

$$
\varepsilon_{\mathrm{F}}=I_{s c}-\phi_{m}-\Delta \phi, \quad \Delta \phi=-4 \pi e^{2} \lambda N_{d} Z_{d},
$$

где $I_{s c}$ и $\chi-$ энергия ионизации и электронное сродство полупроводника, $\phi_{m}$ - работа выхода металла, $\lambda-$ плечо димера, $N_{d}$ - поверхностная концентрация дефектов. Здесь предполагается, что значение $N_{d}$ много меньше концентрации поверхностных атомов металла $N_{\text {met }}^{\text {surf }} \sim 10^{15} \mathrm{~cm}^{-2}$. При этом дефекты можно считать не взаимодействующими друг с другом. Поэтому представление состояний дефектов в виде изолированного локального уровня, а не дефектной подзоны остается в силе. То же можно сказать и о выделенных из подложки поверхностных атомах металла, представляемых как адатомы. При этом мы по-прежнему считаем, что приведенные выше аппроксимации для плотностей состояний металла $\rho_{\text {met }}(\omega)$ и $\rho_{\text {met }}^{\prime}(\omega)$ остаются неизменными.

Высоты барьеров Шоттки на контакте металла с электронным $\left(\Phi_{b}^{n}\right)$ и дырочным $\left(\Phi_{b}^{p}\right)$ полупроводниками равны соответственно

$$
\Phi_{b}^{n}=\phi_{m}-\chi+\Delta \phi, \quad \Phi_{b}^{p}=-\phi_{m}+\chi+E_{g}-\Delta \phi .
$$

Таким образом, решая уравнение (6) с учетом (7), по формуле (8) можно вычислить значения высоты БШ $\Phi_{b}^{n, p}$. В настоящей работе мы, однако, не будем проводить расчеты для конкретных БШ, а рассмотрим простой, но интересный с точки зрения теории частный случай.

Положим для простоты $\varepsilon_{m}=\varepsilon_{d}=\varepsilon$. Такое упрощение задачи вполне допустимо: так, например, для целого ряда полупроводников ( $\mathrm{Si}, \mathrm{Ge}, \mathrm{GaAs}, \mathrm{GaSb}$ ) энергии центров запрещенных зон $E_{c}=\chi+E_{g} / 2 \sim 4.5 \mathrm{eV}\left(\sim \varepsilon_{d}\right)$ относительно уровня вакуума [1], что почти совпадает с работами выхода $\phi\left(\sim \varepsilon_{m}\right)$ таких металлов, как $\mathrm{W}$, $\mathrm{Mo}, \mathrm{Ta}, \mathrm{Nb}, \mathrm{Cu}, \mathrm{Ag}$ [19]. Далее пренебрежем для начала зависимостью $\varepsilon_{\mathrm{F}}$ от $Z_{d i m}$, считая $\left|I_{s c}-\phi_{m}\right| \gg|\Delta \phi|$. Выполнение такого неравенства справедливо при концентрации поверхностных дефектов $N_{d} \sim 10^{12} \mathrm{~cm}^{-2}$, когда при $\lambda \sim 3 \AA$ и $\left|Z_{d i m}\right| \sim 1$ получаем $|\Delta \phi| \sim 0.05 \mathrm{eV}$. Для выбранных нами полупроводников и металлов $\left|I_{s c}-\phi_{m}\right| \sim E_{g} / 2$, так что предполагаемое неравенство выполняется. При таких условиях систему уравнений (6) можно свести к задаче Андерсона о магнитном моменте примеси в металле (см. уравнения (18.95) и (18.96) в [20], где $E, \Delta, U$ и $n_{d \uparrow, \downarrow}$ нужно заменить на $\varepsilon, \Gamma$, $G$ и $n_{m, d}$ ). Аналогично [20] мы представили на рисунке области однородного (UCD, $\left.n_{d}=n_{m}\right)$ и неоднородного $\left(\mathrm{NUCD}, n_{d} \neq n_{m}\right)$ распределения заряда на димере. Найдя в нулевом приближении $(\Delta \phi=0)$ значения чисел заполнения из системы уравнений (6), мы можем затем вычислить поправки к ним, пропорциональные малому параметру $\Delta \phi /\left(I_{s c}-\phi_{m}\right)$.

Следует, однако, отметить, что для политипов карбида кремния предложенное упрощение задачи не работает, 


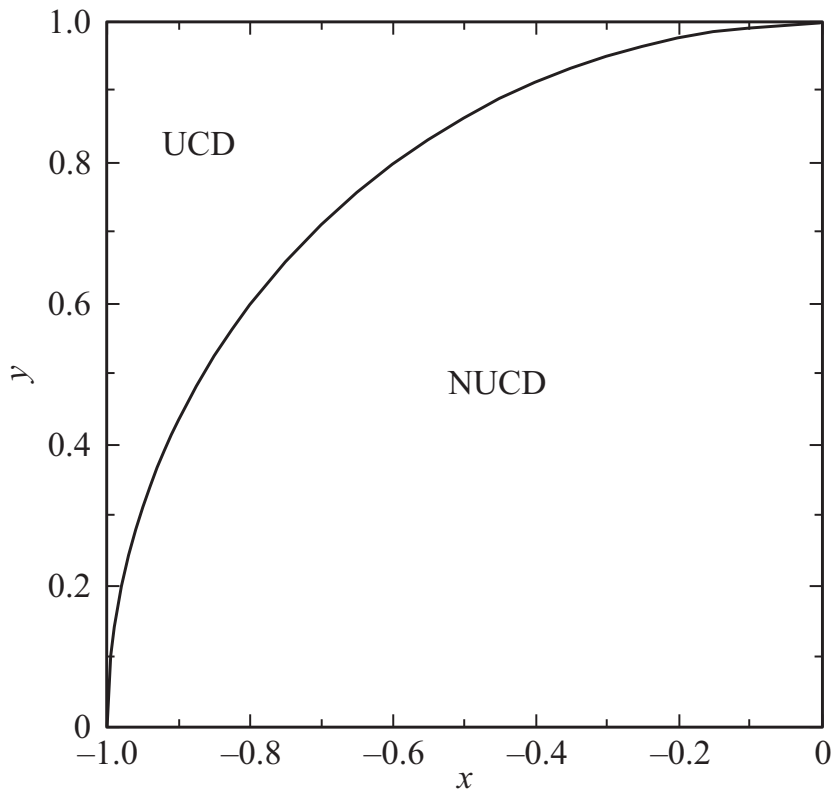

Области однородного (UCD, $\left.n_{d}=n_{m}\right)$ и неоднородного (NUCD, $n_{d} \neq n_{m}$ ) распределения заряда на димере при $\varepsilon_{m}=\varepsilon_{d}=\varepsilon: \quad x=\left(\varepsilon_{\mathrm{F}}-\varepsilon-G / 2\right) / G, y=\pi \Gamma / G$. Сплошной линией представлена граница между областями. Изображена только левая половина диаграммы, симметричной относительно $x=0$.

так как, например, для 4H-SiC концентрация нестехиометрических кремниевых вакансий составляет величину порядка $10^{14} \mathrm{~cm}^{-2}$. В этом случае требуется самосогласованный численный расчет.

\section{Конфликт интересов}

Авторы заявляют, что у них нет конфликта интересов.

\section{Список литературы}

[1] Ф. Бехштедт, Р. Эндерлайн, Поверхности и границы раздела полупроводников (Мир, М., 1990), гл. 4.

[2] W. Mönch, Rep. Prog. Phys., 53 (3), 221 (1990). DOI: $10.1088 / 0034-4885 / 53 / 3 / 001$

[3] R.T. Tung, Appl. Phys. Rev., 1 (1), 011304 (2014). DOI: $10.1063 / 1.4858400$

[4] И.В. Антонова, ФТП, 50 (1), 67 (2016). [Пер. версия: $10.1134 / \mathrm{S} 106378261601005 \mathrm{X} 0]$.

[5] I. Shtepliuk, J. Eriksson, V. Khranovskyy, T. Iakimov, A.I. Spetz, R. Yakimova, Beilstein J. Nanotechnol, 7, 1800 (2016). DOI: 10.3762/bjnano.7.173

[6] H.-M. Chang, K.-L. Fan, A. Charnas, P.D. Ye, Y.-M. Lin, C.-I Wu, C.-H. Wu, J. Phys. D: Appl. Phys., 51 (13), 135306 (2018). DOI: 10.1088/1361-6463/aab063

[7] G.-S. Kim, S.-H. Kim, J. Park, K.H. Han, J. Kim, H.-Y. Yu, ACS Nano, 12 (6), 6292 (2018). DOI: $10.1021 /$ acsnano.8b03331

[8] S. Wang, J.-P. Chou, C. Ren, H. Tian, J. Yu, C. Sun, Y. Xu, M. Sun, Sci. Rep., 9, 5208 (2019).

DOI: $10.1039 / \mathrm{C} 9 \mathrm{CP} 07009 \mathrm{E}$
[9] С.Ю. Давыдов, А.А. Лебедев, О.В. Посредник, Ю.М. Таиров, ФТП, 35 (12), 1437 (2001). [Пер. версия: 10.1134/1.1427974].

[10] С.Ю. Давыдов, ФТТ, 46 (12), 2135 (2004). [Пер. версия: $10.1134 / 1.1841381]$

[11] R. Ludeke, G. Jezequel, A. Taleb-Ibrahimi, Phys. Rev. Lett., 61 (5), 601 (1988). DOI: 10.1103/PhysRevLett.61.601

[12] R. Ludeke, Phys. Rev. B., 40 (3), 1947 (1989). DOI: $10.1103 /$ PhysRevB.40.1947

[13] С.Ю. Давыдов, С.В. Трошин, ФТТ, 49 (8), 1508 (2007). [Пер. версия: 10.1134/S1063783407080318].

[14] С.Ю. Давыдов, А.А. Лебедев, О.В. Посредник, Элементарное введение в теорию наносистем (Лань, СПб., 2014), гл. 8 и 9.

DOI: https://lanbook.fdo.tusur.ru/book/44757? category $=43738$

[15] А.А. Лебедев, ФТП, 33 (7), 769 (1999). [Пер. версия: $10.1134 / 1.1187764]$.

[16] А.И. Гусев, УФН, 184 (9), 905 (2014). DOI: $10.3367 /$ UFNr.0184.201409a.0905

[17] J.K. Freericks, V. Zlatic, Rev. Mod. Phys., 75 (4), 1333 (2003). DOI: 10.1103/RevModPhys.75.1333

[18] В.Ю. Ирхин, Ю.П. Ирхин, Электронная структура, физические свойства и корреляционные эфбекты в $d$ $u$ f-металлах (УрО РАН, Екатеринбург, 2004), гл. 2. DOI: https://www.rfbr.ru/rffi/ru/books/o_71426

[19] Физические величины. Справочник, под ред. И.С. Григорьева, Е.3. Мейлихова (Энергоатомиздат, М., 1991).

[20] Ч. Киттель, Квантовая теория твердых тел (Наука, М., 1967), гл. 18. DOI: https://ikfia.ysn.ru/wp-content/uploads/ 2018/01/Kittel1967ru.pdf 